\title{
Small Angle X-Rays Scattering Studies of Biomolecules
}

\author{
S.S. FUNARI \\ MPI for Colloids and Interfaces, c/o HASYLAB \\ Notkestr. 85, 22603 Hamburg, Germany
}

\begin{abstract}
The building units of biological systems, the biomolecules, cannot easily be organized or even classified into defined categories. They can be as simple as water or complex as tintin, a muscle protein extremely large with several thousand atoms. To understand their function, one must know their characteristics, where they occur and what they do. One approach to reach such an ambitious task is to determine their structure, as single molecules or assembled into aggregates. Small angle X-ray scattering is the most important method for this purpose. We present studies carried out on several systems, and aiming at different questions about them. We start with lipids, the main components of the cell membranes. These membranes form the cell boundaries, the moiety required for the so-called membrane proteins, but also influence significantly several aspects of biological activity. More complex systems like a muscle fibre is also presented, showing that changes in the structure are related to the movement mechanism. It becomes easy to conclude that knowing the structures and the changes occurring in them is an important way to understand the function of biomolecules and therefore their role in the life cycle.
\end{abstract}

PACS numbers: $61.10 .-\mathrm{i}, 87.80 .-\mathrm{y}, 64.75 .+\mathrm{g}$

\section{Introduction}

Since the discovery of X-rays, more than a century ago, science has been making use of it in an ever-growing range of applications, from determination of shoe size to medical diagnosis. Like any radiation, it is scattered by particles, reflecting their characteristics. Proper analysis of this scattered radiation can give information about size, shape, and mass of these particles. For research purposes, tube or rotating anode generators are the common laboratory sources while synchrotron radiation is found only in very large laboratories dedicated to its generation due to the size of the equipment. 
By far the most important aspect of synchrotron radiation in studying biomolecules lies on its high intensity (more precisely, brilliance). This allows data collection in a short time, before the sample's decay. Similar experiments at laboratory sources very often require the exposure time of the sample to the X-ray radiation to be several times longer, which can damage the specimen. The most common source of radiation damage in biomolecules arises from the formation of free radicals after the rupture of double bonds (they are abundant in biomolecules).

Another interesting aspect of the use of the high intensity from synchrotron radiation, and in this case experiments at normal laboratory sources would not give significant results, is the possibility of performing time resolved experiments, joining structural aspects with dynamics and kinetics of different processes. Rapp [1] has previously given a concise review on this topic, showing the wide range of experiments and wealth of information that can be obtained with such measurements.

\section{Methods}

Biomolecules can be studied in many different ways and using several different techniques. Concerning structural aspects we could mention Fourier transform infrared (FTIR), NMR, EPR, fluorescence, electron microscopy, etc. apart from $\mathrm{X}$-rays scattering. Each of them has its advantages, depending on the kind of information being searched. Nowadays, modern scientists have a wide range of methods to pursue their interests, but should also have a broad knowledge of these techniques in order to choose the most appropriate method for each specific question.

Here we give a brief description of the $X$-rays scattering. The systems to be studied, despite being biological or not, can be divided into two main groups: systems with long range order and amorphous systems (or non-ordered systems).

Systems with long range order. In this category we can include crystals (proteins, peptides, sugars, etc.), fibres (such as muscle or collagen) and self-assembled macrodomains of amphiphiles in general. The scattering can be described, at least in a simple manner, by the Bragg law

$$
2 d_{h k l} \sin \theta=\lambda
$$

where $d_{h k l}$ is the scattering plane characterized by the Müller indexes $h k l, 2 \theta$ - the scattering angle and $\lambda$ is the $\mathrm{X}$-rays wavelength of the monochromatic radiation. At the beam line A2 of HASYLAB, $\lambda=0.15 \mathrm{~nm}$.

Amorphous systems, i.e. non-organized independent particles. The angular distribution of the scattered intensity is given by [2]:

$$
I(h)=4 \pi \int_{0}^{\infty} p(r) \frac{\sin (h r)}{h r} \mathrm{~d} r(r),
$$

where $p(r)$ is the distribution function and $h=(4 \pi / \lambda) \sin \theta$. 


\section{Systems}

We present results obtained from a large scope of biomolecules: lipid A, mixtures of phospholipids and non-ionic surfactants, muscles (a very complex fibre structure formed by different molecules, each one with a specific function) and macromolecules (the most common biomacromolecules are proteins).

\subsection{Lipid $A$}

Lipopolysaccharides (LPS) are the major amphiphilic components of the outer leaflet of the outer membrane of Gram-negative bacteria. The hydrophobic portion of LPS, called lipid A, constitutes the chemical minimal structure responsible for biological activity in most test systems and is therefore called the "endotoxic principle" of LPS.

Brandenburg et al. [3] investigated the three-dimensional supramolecular structures and the states of order of the acyl chains of lipid $A$ from different Gram-negative species. They performed small angle X-ray scattering (SAXS) and FTIR spectroscopic measurements at high water content (80-90\%) and different [lipid $\mathrm{A}] /\left[\mathrm{Mg}^{2+}\right]$ molar ratios. Using different sources for the lipid, they observed that parallel to differences in the chemical primary structure, the polymorphism and states of order at $37^{\circ} \mathrm{C}$ of the non-enterobacterial* lipid A were different from those of enterobacterial lipid A. A clear correlation between the supramolecular structure and previously determined biological activities could be established. Lipid A with a strong preference for lamellar structures were endotoxically inactive and lack cytokine-inducing capacity; the compounds assuming a mixed lamellar/non-lamellar structure monophosphoryl (lipid A from S. minesota) are of lower toxicity in vivo, but may induce cytokines in vitro; those lipid A with a strong tendency to form non-lamellar inverted structures (lipid A from S. minesota and Rc. gelatinosus) exhibit full endotoxicity in vitro and in vivo. In contrast, anticomplementary activity is most pronounced for compounds with lamellar and least expressed for those with inverted structures. The states of order at $37^{\circ} \mathrm{C}$ vary non-systematically, exhibiting the highest values for lipid A from $S$. minesota and the lowest for that of Rc. gelatinosus. Based on such results they proposed to change the term endotoxic conformation, which is used to describe the conformation of a single lipid A molecule required for optimal triggering of biological effects, to "endotoxic supramolecular conformation" which denotes the particular organization of lipid A aggregated in physiological fluids causing biological activity. Lipid A obtained from different organisms showed characteristic $\mathrm{X}$-rays scattering patterns at $40^{\circ} \mathrm{C}$, indicating the formation of different structures. Biphosphoryl lipid A from S. minesota showed a cubic (non-lamellar) structure with lattice parameter $a=12.5 \mathrm{~nm}$, however the monophosphoryl showed a much less clear diffraction pattern for the same phase. Lipid A from Rc. gelatinosus

*Enterobacteria is a class of bacteria, many of them causing human diseases, e.g. Salmonella. 
showed a diffraction pattern characteristic of an inverted hexagonal phase with lattice $a=4.24 \mathrm{~nm}$. Interestingly, they observed the formation of two lamellar phases, $a=6.57$ and $a=4.46 \mathrm{~nm}$, when studying lipid A from $R p$. viridis.

Compiling these results together with that from FTIR spectroscopy and other techniques they were able to establish a correlation between self-assembled supramolecular structures with biological activity of different lipid A preparations, summarized in Fig. 1.

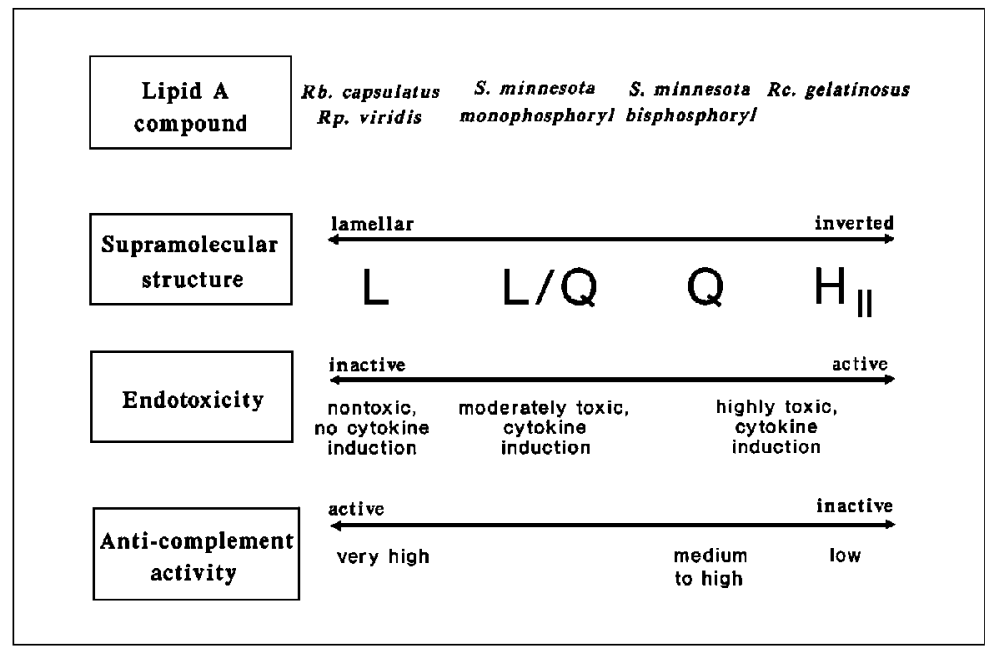

Fig. 1. Schematic diagram of the correlation between supramolecular structure and biological activity of different lipid A preparations. Toxicity includes lethality and pyrogenicity in mice and rabbits, respectively, and cytokine induction relates to tumor necrosis factor production in mouse macrophages. Anticomplement activity was determined by incubation of free lipid A with guinea pig serum as complement source and subsequent inhibition of sheep erythrocyte lysis. $\mathrm{L}-$ lamellar, $\mathrm{Q}-$ cubic, $\mathrm{H}_{\mathrm{II}}$ - inverted hexagonal phases. The very high anticomplement activity only refers to $R p$. viridis, while medium to high activity includes also synthetic lipid A from E. coli (from Ref. [3], reprinted with permission from Blackwell Publishing).

An extension of these studies led to investigations of the behaviour of the acyl chains and their influence on biological activity of endotoxins, monitored by the wide-angle (WAXS) region of the scattering pattern [4], Fig. 2. The lipids investigated differed in the length of the sugar portion and various saturated and unsaturated phospholipids with different head groups under near a physiological water content $(>85 \%)$. It was observed that the packing density of the saturated endotoxin acyl chains is lower than those of saturated phospholipids but similar to those of monounsaturated phospholipids, each in the gel phase. The hydrophobic moiety of endotoxins thus exhibits significant conformation disorder already in the gel phase. The acyl chain packing of the endotoxins decreases with increasing 


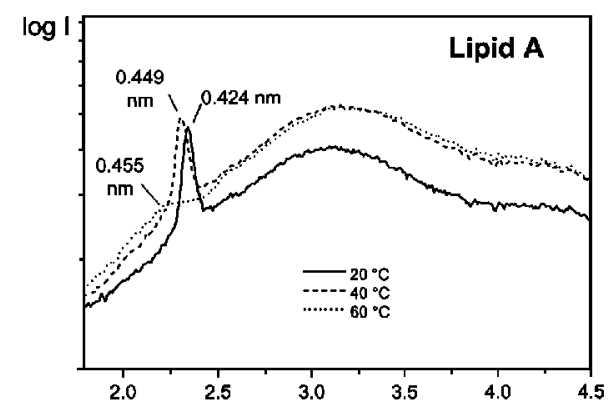

Fig. 2. Wide-angle X-ray scattering patterns of lipid A at $85 \%$ buffer content at different temperatures. The formation of a gel $\mathrm{L}$ phase is clearly seen as well as the change in the lattice parameter as the temperature changes [4].

length of the sugar chain, which seems to be relevant to the observed differences in biological activity.

\subsection{Lipid-surfactant mixtures}

Membrane solubilization in surfactants is a key technique in biochemistry. However, the properties of mixed detergent/lipid micelles only came into focus with the discovery of detergent insoluble regions (called rafts) in cell membranes [5].

Mixtures of the non-ionic detergent ${ }^{\dagger} \mathrm{C}_{12} \mathrm{EO}_{8}$ with disaturated phospholipids such as DMPC or DPPC in water produced an unusual micelle. These mixtures undergo a structural transition upon cooling through the chain melting temperatures of the respective phospholipids, resulting in the formation of mixed micelles [6]. The translucent micellar solutions showed characteristic wide angle $\mathrm{X}$-ray scattering that were attributed to ordered hydrocarbon chains, whereas the absence of small angle $\mathrm{X}$-ray reflections indicated that there is no long range order in these mixtures, see Fig. 3. The presence of ordered phospholipid acyl chains was confirmed by differential scanning calorimetry (DSC) and isothermal titration calorimetry (ITC). The endothermic DSC signals observed in the upscan mode were tentatively ascribed to chain melting and mixing of the components. Isothermal titration of the mixed-micellar solutions into an excess of the detergent $\mathrm{C}_{12} \mathrm{E} \mathrm{O}_{8}$ resulted in sudden uptake of the latent heat by the gel state phospholipids. The heat uptake per mol of phospholipid decreased with increasing detergent/phospholipid molar ratio. A simple geometric model is presented in Fig. 4, assuming that the dominating particle species in the mixtures is a discoidal phospholipid aggregate with ordered acyl chains, surrounded by a toroidal detergent hoop. The model described implies that the fraction of ordered phospholipid chains decreases with increasing detergent/phospholipid molar ratio, in agreement with the calorimetric results and with high resolution NMR spectroscopy.

${ }^{\dagger} \mathrm{C}_{12} \mathrm{EO}_{8}$ - octaethyleneglycol-mono- $n$-dodecylether, DMPC - 1,2-dimyristoyl-sn-glycero-3-phosphocholine and DPPC - 1,2-dipalmitoyl-sn-glycero-3-phosphocholine. 

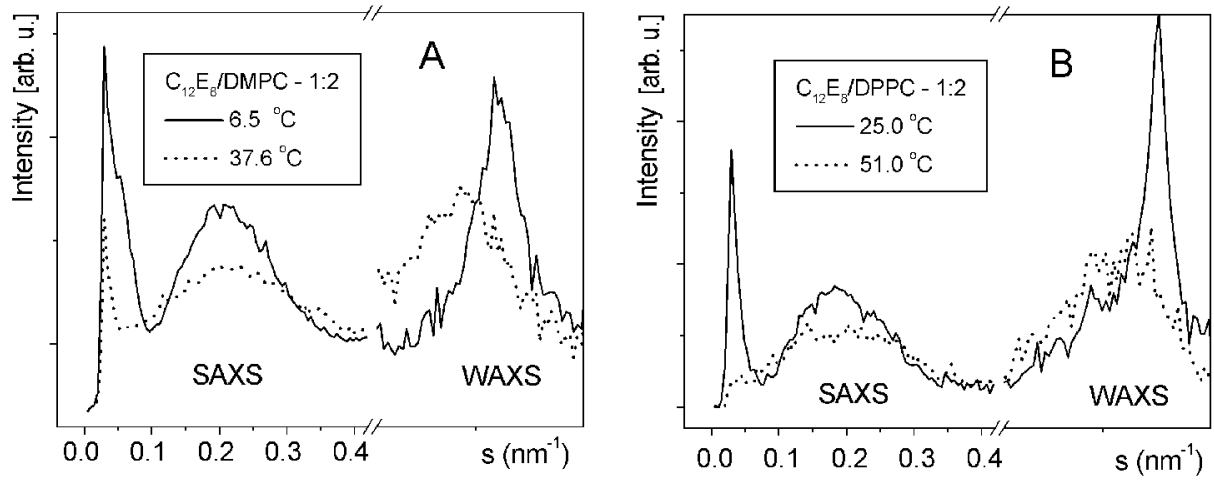

Fig. 3. Synchrotron X-ray patterns of (A) $\mathrm{C}_{12} \mathrm{E}_{8} / \mathrm{DMPC}$ and (B) $\mathrm{C}_{12} \mathrm{E}_{8} / \mathrm{DPPC}$ mixtures at molar ratio 1:2 obtained below and above the respective phase transition temperatures of the pure phospholipids [5]. Copyright (2001) National Academy of Sciences, U.S.A.

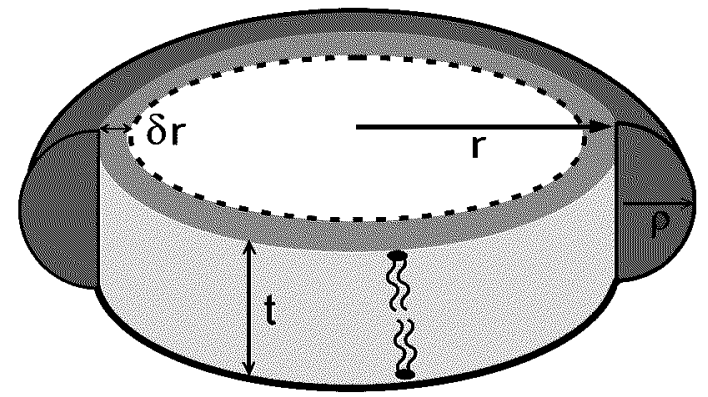

Fig. 4. Model of the gel-state micelle. $t$ - thickness of the phospholipid bilayer, $r$ radius of the bilayer patch, $\delta r$ - width of the perturbed lipid bilayer, $\rho$ - radius of the $\mathrm{C}_{12} \mathrm{EO}_{8}$ semitorus [5]. Copyright (2001) National Academy of Sciences, U.S.A.

Studies on the POPC/ $\mathrm{C}_{12} \mathrm{EO}_{2} /{ }^{2} \mathrm{H}_{2} \mathrm{O}$ system at $25^{\circ} \mathrm{C}$ showed the possibility of modifying the curvature of lipid bilayers [7, 8]. To determine the evolution of the geometrical parameters with composition, X-ray diffraction patterns were collected as a function of the relative humidity, $\mathrm{RH}$. The formation of an unexpected hexagonal phase indicated a peculiar behaviour in these mixtures, considering that neither the lipid nor the surfactant form such phase in aqueous dispersions.

Samples partially hydrated, RH $<0.4$ showed that at low lipid contents, $R_{\mathrm{s} / 1}<0.5$ a lamellar gel phase was formed, changing to hexagonal as the surfactant ratio increases. The cylinder radius of the $H_{I I}$ phase is considerably smaller than that previously observed in cubic phases of the same system. Moreover, comparing the variation of the repeat distance, $d$, of the gel phase with $\mathrm{RH}$ (or the content of water) we have been able to show that the uptake of water by the $L_{\beta}$ gel phase is higher $\partial d / \partial R H=60.1 \mathrm{~nm} / \mathrm{RH}$-unit as a single phase than in the $L_{\beta}+\mathrm{H}_{\mathrm{II}}$ two 
phase region $\partial d / \partial R H=57.1 \mathrm{~nm} / \mathrm{RH}$-unit. The water content is important for the stabilization of $\mathrm{H}_{\mathrm{II}}$ phase and determination of its characteristic dimensions. However, it was argued that the interaction between the surfactant and the lipid is the key factor for $\mathrm{H}_{\mathrm{II}}$ phase formation and that the $\mathrm{EO}_{2}$ head groups displace water from the inner parts of the polar region of the mesogenic units.

The same system, at high water contents, has also shown the formation of cubic phases related by an epitaxial relationship and fulfilling the conditions of a Bonnet transformation [9].

\subsection{Muscle}

A host of essential intracellular processes depend on transduction of chemical free energy into mechanical work by isoforms of the molecular motor, myosin. The S1 (subfragment 1, the myosin head) moiety of myosin consists of a motor domain (bearing actin and ATP binding sites) and a tail domain (bearing the essential and regulatory light chains). At least 17 classes of myosin exist, having similar motor domain structures, but diverging extensively in other domains to reflect their differing functions. Their similarity in motor domains suggests that the mechanism of myosin motors, and probably also of the kinesin motor family, is fundamentally similar. For motility studies, myosin II, the myosin class responsible for contractility in muscle, possesses the unique advantage that, in striated muscle cells, it is aggregated into highly ordered filaments, which are themselves orientated and positioned in a quasi-crystalline manner. This allows both structural and mechanical data to be collected simultaneously from a population of working S1 moieties, which is presently impossible for other myosin classes or from crystallographic preparations of myosin II.

Since 1969, the proposed mechanism of S1 work production has been rotational displacement of some portion of $\mathrm{S} 1$ about a point in the actomyosin complex (the power stroke), producing an unloaded filament translation of $c a .10 \mathrm{~nm}$ or an isometric force of $1-5 \mathrm{pN}$. Attempts to detect this rotation in muscle by fluorescence polarisation or electron spin probes were initially unsuccessful because probes were located on the motor domain of S1, which is now thought not to rotate. However, crystallographic studies of S1 showed three S1 structural states, depending on the nucleotide occupancy of the S1 active site, which are consistent with a tail rotation power stroke. The relation between tail orientation and S1 force is crucial to a quantitative description of the S1 motor, but it is not possible to associate a level of S1 force development with in vitro crystal structures, and it is of great importance to demonstrate similar S1 structural changes in situ, from working myosin molecules. Time-resolved X-ray diffraction studies on myosin II in intact muscle fibres provide a non-invasive method of detecting S1 structural states in situ and relating them to the level of S1 force development.

The X-ray pattern from striated muscle is divisible into equatorial and meridional reflections. Equatorial reflections are intense reflections arising from 
radial mass distribution within the myofilament lattice, used to detect the radial transfer of S1 mass during cross-bridge formation and changes in filament separation [10]. Meridional reflections are an order of magnitude less intense, arising from the axial mass projection of the sarcomere, some of which show intensity changes accompanying a synchronised power stroke. On X-ray pattern also off-meridional layer lines appear, which detect changes in the inherent helical structure of both the thick and thin filaments, including the structural changes in the regulatory proteins which accompany calcium activation. The meridional reflection at $14.5 \mathrm{~nm}$ in contracting muscle (M3) corresponds to the third harmonic of the axial unit cell of the myosin filament. Its intensity $\left(I_{\mathrm{M} 3}\right)$ depends on the sampling of the transform of S1 structure by this reflection, so $I_{\mathrm{M} 3}$ is an important monitor of S1 structure.

$I_{\mathrm{M} 3}$ falls in response to both stretches and releases, but for a release there is a small delay before the intensity fall, which is absent for stretches. This has been interpreted as resulting from two components which form the $I_{\mathrm{M} 3}$ response to length changes; an elastic S1 distortion, and an active, power stroke distortion, both of which cause a bending of the tail domain. The instantaneous fall in intensity for a stretch occurs because the elastic displacement accompanying the length fall is merged with the power stroke intensity fall, which follows after the length change is complete. But for a release, the tail domain bending passes through a maximum value of $I_{\mathrm{M} 3}$ before falling, which causes a delay in the $I_{\mathrm{M} 3}$ fall for releases [11]. The proposal that elastic and active tail displacements sum to produce the $I_{\mathrm{M} 3}$ signal for a length change is supported by data from sinusoidal oscillations of muscle length at different temperatures. Bagni et al. [12] showed that for small
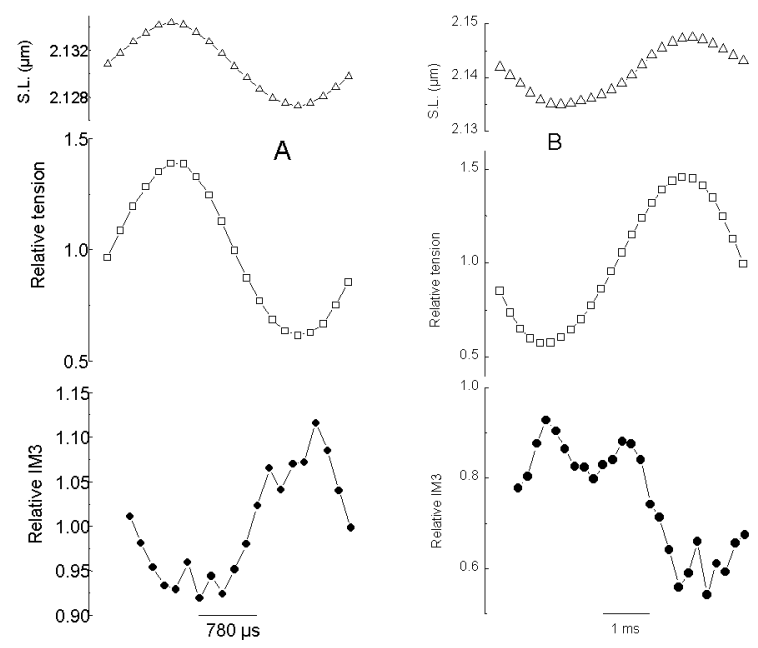

Fig. 5. $\quad I_{\mathrm{M} 3}$ signals for sinusoidal length oscillations at $3 \mathrm{kHz}$ (A) and $200 \mathrm{~Hz}$ (B) [12]. Note the distortion of $I_{\mathrm{M} 3}$ during the release phase of the sinusoid at $200 \mathrm{~Hz}$, forming a double peak, which is absent at $3 \mathrm{kHz}$ (reprinted with permission from Biophysical Society). 
amplitude sinusoidal length oscillations ( $2.5 \mathrm{~nm}$ per half sarcomere) at frequencies $1 \mathrm{kHz}, I_{\mathrm{M} 3}$ changes were roughly sinusoidal, but at frequencies $<1 \mathrm{kHz}, I_{\mathrm{M} 3}$ was increasingly distorted, Fig. 5. The power stroke kinetics act in the millisecond time domain, hence at low frequencies, where power stroke kinetics are fast enough to produce significant active displacement of the tail, total tail movement would be greater than at high frequencies, where tail displacement would be almost entirely elastic. The isometric tail position lies close to its position at maximum $I_{\mathrm{M} 3}$; during the release phase of the sinusoids (i.e. fibre length less than rest length), the tail moves towards its position at maximum $I_{\mathrm{M} 3}$ [13]. The combined displacement at low frequencies was great enough to carry the tail domain through its $I_{\text {M3max }}$ position during the release phase, causing an intensity double peak at maximum shortening, while at high frequencies, elastic tail displacement was insufficient to reach maximum $I_{\text {M3 }}$.

\subsection{Macromolecules}

The X-rays scattering pattern from dilute solutions of macromolecules or particles shows no peaks (Bragg interference), because supposedly there are no interactions between independent particles. Therefore, the total scattering is the sum of contributions from single particles only. Synchrotron radiation X-ray scattering is an established method to monitor low resolution structure of (bio)-macromolecules [14]. Modern methods of analysis are capable of $a b$ initio retrieving the particle characteristics $[15,16]$. A nice example is found in the determination of the shape and size of 5S rRNA [17], an important part of the ribosome.

Ribosomal 5S rRNA is approximately 120 nucleotides long and is an essential component of the ribosome. Ribosomal particles lacking $5 \mathrm{~S}$ rRNA have a strongly reduced activity in protein synthesis [18], in particular, the peptidyl transferase activity is greatly reduced. Because of its functional importance and of the fact that the $5 \mathrm{~S}$ rRNA interacts specifically with several ribosomal, it is of great interest to know the three-dimensional structure or a reliable shape of this RNA molecule. Recently, these new approaches $[15,16]$ have been applied to ab initio restoring a model of the free $5 \mathrm{~S}$ rRNA in solution, derived from SAXS data.

The composite $\mathrm{X}$-rays solution scattering curve from the $5 \mathrm{~S}$ rRNA extrapolated to zero concentration is presented in Fig. 6, curve 1. The maximum dimension and the radius of gyration of the particle are $12.0 \pm 0.5 \mathrm{~nm}$ and $3.44 \pm 0.05 \mathrm{~nm}$, respectively. The calculated distance distribution function $p(r)$ is typical of an elongated particle. The "shape scattering" curve, after a constant subtraction (Fig. 6, curve 2) yields the Porod volume of $37 \pm 2 \mathrm{~nm}^{3}$. This volume corresponds well to the dry volume of the $5 \mathrm{~S}$ RNA $\left(38.8 \mathrm{~nm}^{3}\right)$ computed from its molecular mass assuming the partial specific volume of $0.53 \mathrm{~cm}^{3} / \mathrm{g}$.

The ab initio shape of the free $5 \mathrm{~S}$ rRNA in solution in Fig. 7 is much more anisometric than the fragment of the rRNA in the 50S ribosomal subunit E. Coli complexed with three protein globules and identified as the $5 \mathrm{~S}$ rRNA in the map 


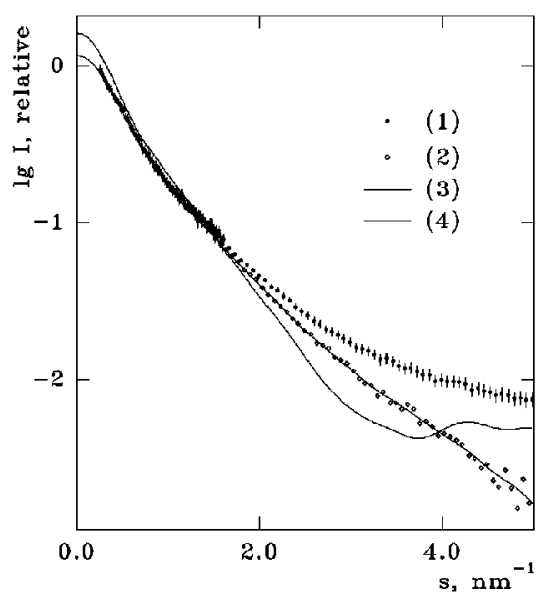

Fig. 6. X-ray scattering of $5 \mathrm{~S}$ rRNA [17] in solution and calculated from different models. 1 - composite experimental curve, 2 - shape scattering curve after subtraction of a constant, 3 - scattering from $a b$ initio dummy atom model, 4 - scattering from the model of Westof et al. [20].
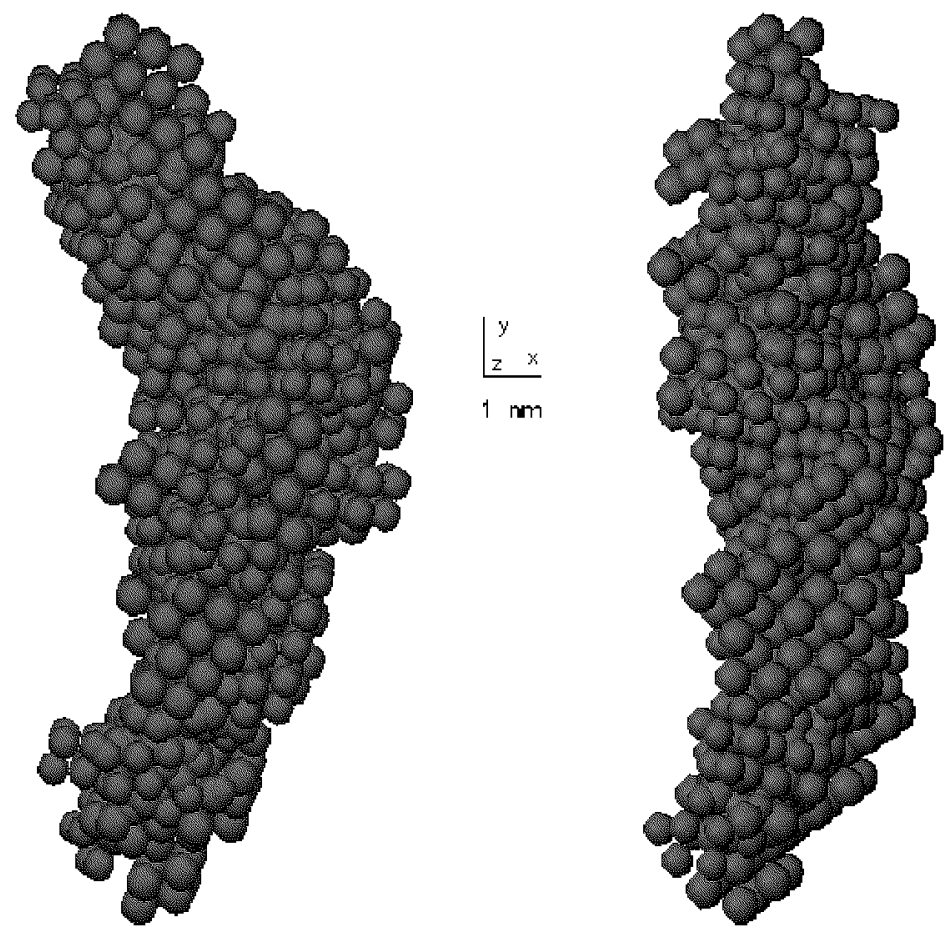

Fig. 7. Superposition of 10 low resolution models of the 5S rRNA, from SAXS data [17]. Reprinted with the permission from American Society for Biochemistry and Molecular Biology. 
of Svergun and Nierhaus [19]. This provides direct evidence that the 5S rRNA becomes essentially more compact during the complex formation with the ribosomal proteins.

The obtained model shape should help to construct a high resolution three-dimensional model of the complete native 5S rRNA and may be later on also applied for crystallographic molecular replacement studies, as well as in the analysis of the complex formation with binding proteins in solution.

\section{Conclusions}

The different studies presented have shown that SAXS not only is important to determine the structural aspects, but also gives information that can be linked to the dynamic and functional properties of a system. This was evidenced on muscle studies and using lipid $A$, where a relationship between structure (that is dependent on the preparation) and biological activity has been established. Different mesogenic units can be obtained by mixtures of lipids and detergents, allowing one to control the shape and curvature of the lipid bilayer to be formed in a model system. Moreover, it could be shown that hydration of different phases forming a two-phase system is not equal, but rather occurs preferentially in one of the phases present.

Finally, the possibility to obtain the structure, even though at low resolution, of a protein is a significant contribution to our understanding of this class of biomolecules. The structure obtained showed that the $5 \mathrm{~S}$ during the complex formation with the ribosomal proteins rRNA becomes more compact. Changes in a protein due to interactions between protein-receptor should soon become "visible" with the aid of modern methods to retrieve structural characteristic parameter of a scattering particle.

\section{Acknowledgments}

I thank P.J. Griffiths and G. Rapp for their support and help, in particular with the muscle part, in which they are the experts.

\section{References}

[1] G. Rapp, Acta Phys. Pol. A 82, 103 (1992).

[2] I. Pilz, O. Glatter, O. Kratky, Methods in Enzymology 61, 148 (1979).

[3] K. Brandenburg, H. Mayer, M.H.J. Koch, J. Weckesser, E.Th. Rietschel, U. Seydel, Eur. J. Biochem. 218, 555 (1993).

[4] K. Brandenburg, S.S. Funari, M.H.J. Koch, U. Seydel, J. Struct. Biol. 128, 175 (1999).

[5] S.S. Funari, B. Nuscher, G. Rapp, K. Beyer, Proc. Natl. Acad. Sci. 98, 8938 (2001). 
[6] D. Otten, L. Löbbecke, K. Beyer, Biophys. J. 68, 584 (1995).

[7] S.S. Funari, C. di Vita, G. Rapp, Acta Phys. Pol. A 91, 953 (1997)

[8] S.S. Funari, Eur. Biophys. J. 27, 590 (1998).

[9] S.S. Funari, B. Mädler, G. Rapp, Eur. Biophys. J. 24, 293 (1996).

[10] M.A. Bagni, G. Cecchi, P.J. Griffiths, Y. Maeda, G. Rapp, C.C. Ashley, Biophys. J. 67, 1965 (1994).

[11] V. Lombardi, G. Piazzesi, M.A. Ferenczi, H. Thirlwell, I. Dobbie, M. Irving, Nature 374, 553 (1995)

[12] M.A. Bagni, B. Colombini, H. Amenitsch, S. Bernstorff, C.C. Ashley, G. Rapp, P.J. Griffiths, Biophys. J. 80, 2809 (2001)

[13] G. Piazzesi, V. Lombardi, M.A. Ferenczi, H. Thirlwell, I. Dobbie, M. Irving, Biophys J. 68, Suppl, 92S (1995).

[14] L.A. Feigin, D.I. Svergun, Structure Analysis by Small-Angle X-ray and Neutron Scattering, Plenum Press, New York 1987.

[15] D.I. Svergun, V.V. Volkov, M.B. Kozin, H.B. Stuhrmann, Acta Crystallogr. A 52, 419 (1996)

[16] D.I. Svergun, Biophys. J. 76, 2879 (1999).

[17] S.S. Funari, G. Rapp, M. Perbrandt, K. Dierks, M. Vallazza, C. Betzel, V.A. Erdmann, D.I. Svergun, J. Biol. Chem. 275, 31283 (2000).

[18] R.K. Hartmann, D.W. Vogel, R.T. Walker, V.A. Erdmann, Nucleic Acids Res. 16, 3511 (1988).

[19] D.I. Svergun, K.H. Nierhaus, J. Biol. Chem. 275, 14432 (2000).

[20] E. Westhof, P. Romby, P.J. Romaniuk, J.P. Ebel, C. Ehresmann, B. Ehresmann, J. Mol. Biol. 207, 417 (1989). 